\title{
A Study on the Conversion of a Semigroup to a Semilattice
}

\author{
Bahman Tabatabaie, Seyed Mostafa Zebarjad \\ Department of Mathematics, Shiraz University, Shiraz, Iran \\ E-mail: s_zebarjad@yahoo.com \\ Received January 19, 2011; revised March 15, 2011; accepted March 25, 2011
}

\begin{abstract}
The main aim of the current research has been concentrated to clarify the condition for converting the inverse semigroups such as $S$ to a semilattice. For this purpose a property the so-called $E^{*}$ - unitary has been defined and it has been tried to prove that each inverse semigroups limited with $E^{*}$-unitary show the specification of a semilattice.
\end{abstract}

Keywords: Semigroup, Semilattice, $E^{*}$ - unitary

\section{Introduction}

\subsection{Literature Survey}

Literature survey done by the authors show that a special class of semigroups possessing is formed by the $E^{*}-$ unitary inverse semigroups, sometimes also called $0-$ $E^{*}$ - unitary , which was defined by Szendrei [1] and has been intensely studied in the semigroup literature. See, for example, Kellendonk's topological groupoid is Hausdorff when $S$ is $E^{*}$ - unitary [2], and the related class of $E$ - unitary inverse semigroups have also been shown to provide Hausdorff groupoids [3]. In the current research the authors try to prove that each inverse semigroups limited with $E^{*}$ - unitary show the specification of a semilattice. For this purpose, firstly we present elementary concepts as follows.

\subsection{Preliminary Definitions and Propositions}

A groupoid is a set $G$ together with a subset $G^{2} \subseteq G \times G$, a product map $(a, b) \mapsto a b$.

From $G^{2}$ to $G$, and an inverse map $a \mapsto a^{-1}$ (so that $\left.\left(a^{-1}\right)^{-1}=a\right)$ from $G$ onto $G$ such that:

1) if $(a, b),(b, c) \in G^{2}$, then $(a b, c),(a, b c) \in G^{2}$ and $(a b) c=a(b c)$.

2) $\left(b, b^{-1}\right) \in G^{2}$ for all $b \in G$, and if $(a, b) \in G^{2}$ then $a^{-1}(a b)=b$ and $(a b) b^{-1}=a$.

Note that $G^{2}$ is nothing but the set of all pairs $(x, y)$ in $G \times G$ for which $x y$ is defined, and $G^{2}$ is called the set of composable pairs of the groupoid $G$ [3].

If $x \in G, d(x)=x^{-1} x$ is the domain of $x$ and $r(x)=x x^{-1}$ is its range. The pair $(x, y)$ is composable if and only if the range of $y$ is the domain of $x$. $G^{0}=d(G)=r(G)$ is the unit space of $G$, its elements are units in sense that $x \mathrm{~d}(x)=x$ and $r(x)=x$ [4].

By an inverse semigroup we mean a semigroup $S$ such that for each $a$ in $S$, there exists a unique element $a^{*}$ in $S$ with the following properties:

$$
a a^{*} a=a \text {, and } a^{*} a a^{*}=a^{*}
$$

It is well known that the correspondence $a \mapsto a^{*}$ is an involutive anti-homomorphism, i.e., $(a b)^{*}=b^{*} a^{*}$ for all $a$ and $b$ in $S$. It is very common to denote it by $E(S)$, the set of all idempotent elements of $S$, it means that $a^{2}=a$ for all $a$ in $E(S)$. It is clear that $a^{*}=a$ for all $a$ in $E(S)$.

A very important example of an inverse semigroup is given by $S=I(X)$ the set of all partial one-to-one maps on a set $X$. So each element of $I(X)$ is a bijection form a subset $U$ of $X$ onto another subset $V$ of $X$. The set $I(X)$ is a semigroup where the multiplication rule is given by composition of partial maps with the largest possible domain.

For example, if $\theta_{1}, \theta_{2} \in I(X)$ with $\theta_{1}: U_{1} \rightarrow V_{1}$ and $\theta_{2}: U_{2} \rightarrow V_{2}$, then

$$
\theta_{1} \theta_{2}: \theta_{2}^{-1}\left(V_{2} \cap U_{1}\right) \rightarrow \theta_{1}\left(V_{2} \cap U_{1}\right)
$$

is given by:

$$
\theta_{1} \theta_{2}(a)=\theta_{1}\left(\theta_{2}(a)\right)
$$


The element $\theta_{1}^{*}$ is taken to be $\theta_{1}^{-1}$. It is easily checked that $I(X)$ is an inverse semigroup [3,5].

We recall that a relation $\leq$ on a set $X$ is called a partial ordering of $X$ if for all $a, b, c \in X$ :

$$
\begin{aligned}
& \text { 1) } a \leq a \\
& \text { 2) } a \leq b \text { and } b \leq a \text { implies } a=b \\
& \text { 3) } a \leq b \text { and } b \leq c \text { implies } a \leq c \text {. }
\end{aligned}
$$

The following example is of great importance to us. Define $e \leq f(e, f \in E(S))$ to mean $e f=f e=e$. It is clear that $\leq$ is a partial ordering of $E(S)$. We shall call $\leq$ the natural partial ordering of $E(S)$.

An element $b$ of a partially ordered set $X$ is called an upper bound of a subset $Y$ of $X$, if $y \leq b$ for each $y$ in $Y$. An upper bound $b$ of $Y$ is called a least upper bound or join of $Y$, if $b \leq c$ for every upper bound $c$ of $Y$. If $Y$ has a join in $X$, it is clearly unique. Lower bound and greatest lower bound or meet can be defined similarly.

A partially ordered set $X$ is called a semilattice if every two elements subset $\{a, b\}$ of $X$ has a join and a meet in $X$; it implies that every finite subset of $X$ has both a join and a meet. The join (or meet) of $\{a, b\}$ will be denoted by $a \wedge b$ (or $a \vee b)$ [3].

Definition 1.1 Suppose that $S$ is an inverse semigroup and $X$ can be assumed that as a locally compact Hausdorff topological space.

An action of $S$ on $X$ is a semigroup homomorphism as follows:

$$
\begin{aligned}
\alpha: S & \rightarrow I(X) \\
a & \mapsto \alpha_{a}
\end{aligned}
$$

such that

1) for every $a \in S$ there is a continuous $\alpha_{a}$ with open domain in $X$.

2) the union of the domains of all the $\alpha_{a}$ coincides with $X$.

Proposition 1.2 Let $S$ be an inverse semigroup, $\alpha$ an action of $S$ on a set $X$ and $a \in S$, then

$$
\alpha_{a} \alpha_{a^{*}} \alpha_{a}=\alpha_{a} \text { and } \alpha_{a^{*}} \alpha_{a} \alpha_{a^{*}}=\alpha_{a^{*}}
$$

Proof: Since $\alpha$ is an action of $S$ on $X$ then $\alpha: S \rightarrow I(X)$ is a semigroup homomorphism, so for every $a \in S$ we have $\alpha(a) \alpha\left(a^{*}\right) \alpha(a)=\alpha(a)$, then $\alpha_{a} \alpha_{a^{*}} \alpha_{a}=\alpha_{a}$, and simillary $\alpha_{a^{*}} \alpha_{a} \alpha_{a^{*}}=\alpha_{a^{*}}$

With regard to the above text one may conclude that, $\alpha_{a^{*}}=\alpha_{a}^{-1}$, and if $e \in E(S)$, so $\alpha_{e}$ is the identity map on its domain.

Since the range of each $\alpha_{a}$ coincides with the domain of $\alpha_{a^{*}}=\alpha_{a}^{-1}$, therefore it can be open as well as its domain. Also it can be mentioned that $\alpha_{a}^{-1}$, is continu- ous, so $\alpha_{a}$ is necessarily a homeomorphism onto its range.

For every $e \in E(S)$ the domain (and range) of $\alpha_{e}$ can be denoted by $E_{e}$, it means:

$$
\alpha_{e}: E_{e} \rightarrow E_{e} \text {. }
$$

It is clear to show that the domains of both $\alpha_{a}$ and $\alpha_{a^{*} a}$ is the same, and implies that the domain of $\alpha_{a}$ is $E_{a^{*} a}^{a^{*} a}$. Likewise the range of $\alpha_{a}$ is given by $E_{a a^{*}}$. Thus $\alpha_{a}: E_{a^{*} a} \rightarrow E_{a a^{*}}$ is a homeomorphism for every $a \in S$. Briefly if $e^{a}$ and $f$ are in $E(S)$ then we have $\alpha_{e} \alpha_{f}=\alpha_{e f}$ and $E_{e} \cap E_{f}=E_{e f}$.

Proposition 1.3 For each $a \in S$ and $e \in E(S)$ we have: $\alpha_{a}\left(E_{e} \cap E_{a a^{*}}\right)=E_{a e a^{*}}$

Proof: Since N. Sieben [6], R. Exel [7] and Lawson [8] proved it, the authors use their result.

Definition 1.4 Let $\sum$ be the subset of $S \times X$ given by:

$$
\Sigma=\left\{(a b) \in S \times X: b \in E_{a^{*} a}\right\}
$$

and for every $\left(a_{1}, b_{1}\right)$ and $\left(a_{2}, b_{2}\right)$ in $\sum$ we will say that $\left(a_{1}, b_{1}\right) \sim\left(a_{2}, b_{2}\right)$ if $b_{1}=b_{2}$ and there exists an idempotent $e$ in $E(S)$ such that $b_{1} \in E_{e}$, and $a_{1} e=a_{2} e$.

It is clearly that the relation $\sim$ is an equivalence relation on $\Sigma$ The equivalence class of $(a, b)$ will be denoted by $[a, b]$.

Let $G=\{[a, b]: a \in S, b \in X\}$ and put

$$
G^{2}=\left\{\left(\left[a_{1}, b_{1}\right],\left[a_{2}, b_{2}\right]\right) \in G \times G: b_{1}=\alpha_{a_{2}}\left(b_{2}\right)\right\}
$$

And for every $\left(\left[a_{1}, b_{1}\right],\left[a_{2}, b_{2}\right]\right) \in G^{2}$ define:

$$
\left\{\begin{array}{l}
{\left[a_{1}, b_{1}\right] \cdot\left[a_{2}, b_{2}\right]=\left[a_{1} a_{2}, b_{2}\right]} \\
{\left[a_{1}, b_{1}\right]^{-1}=\left[a_{1}^{*}, \alpha_{a_{1}}\left(b_{1}\right)\right]}
\end{array}\right.
$$

it is easy to see that $G$ is a groupoid [3] and the unit space $G^{(0)}$ of $G$ naturally identifies with $X$ under the correspondence

$$
[e, b] \in G^{(0)} \mapsto b \in X,
$$

where $e$ is any idempotent such that $e \in E_{S}$. We show $G$ semigroup as $G(\alpha, S, X)$.

We would now like to give $G$ is a topology. Let $a \in S$ and $U$ be an open subset of $E_{a^{*} a}$ we define $\psi(a, U)$ as follows:

$$
\psi(a, U)=\{[a, b] \in G: b \in U\}
$$

The collection of all $\psi(a, U)$ is the basis of a topology on $G$, and also the multiplication and inversion operations on $G$ are continuous, therefore $G$ is a topological groupoid. 


\section{Main Results}

Recall from [2] that an inverse semigroup $S$ is naturally equipped with a partial order defined by:

$$
a \leq b \leftrightarrow a=b a^{*} a \forall a \in S
$$

Proposition 2.1 Assume that $S$ is an inverse semigroup which is a semilattice. Suppose that $\alpha$ is an action of $S$ on a locally compact Hausdorff space $X$, such that for each $a \in S$, the domain $E_{a^{*} a}$ of $\alpha_{a}$ is closed. Then $G=G(\alpha, S, X)$ is Hausdorff.

Proof:Suppose $[a, c]$ and $[b, d]$ are two distinct elements of $G(\alpha, S, X)$. The aim is to find two disjoint open subsets $T_{1}$ and $T_{2}$ of $G(\alpha, S, X)$ such that:

$$
[a, c] \in T_{1},[b, d] \in T_{2}, T_{1} \cap T_{2}=\phi
$$

We consider two cases:

Case 1): If $(c \neq d)$ :

Since $X$ is Hausdorff space then

$$
\exists F_{1}, F_{2} \subseteq X \text { (open) }, c \in F_{1}, d \in F_{2}, F_{1} \cap F_{2}=\phi
$$

Now let $T_{1}=\psi\left(a, F_{1} \cap E_{a^{*} a}\right)$ and $T_{2}=\psi\left(b, F_{2} \cap E_{b^{*} b}\right)$

Since $T_{1}$ and $T_{2}$ are open set and

$$
\begin{aligned}
& T_{1}=\left\{[a, k] \in G: k \in F_{1} \cap E_{a^{*} a}\right\}, \\
& T_{2}=\left\{[b, k] \in G: k \in F_{2} \cap E_{b^{*} b}\right\},
\end{aligned}
$$

It is clearly that:

$$
[a, c] \in T_{1},[b, d] \in T_{2} \text { and } T_{1} \cap T_{2}=\phi
$$

Case 2): If $(c=d)$ :

Since $S$ is a semilattice let $h=a \wedge b$ so

$$
\left\{\begin{array}{l}
h \leq a \rightarrow h=a h^{*} h \\
h \leq b \rightarrow h=b h^{*} h
\end{array} \Rightarrow[a, c]=[b, c]\right.
$$

Then referring to what proposed in Definition 1.4. $c \notin E_{h^{*} h}$. But $E_{h^{*} h}$ is closed then $T_{2}=X \backslash E_{h^{*} h}$ can be open and $c \in T_{2}$.

Now we can set $T$ as $T_{2} \cap E_{a^{*} a} \cap E_{b^{*} b}$. But we know that $\psi(a, T)=\{[a, k]: k \in T\} \stackrel{a^{*} a}{\text { and }}$ it is clear that $[a, c] \in \psi(a, T),[b, c] \in \psi(b, T)$.

To do so it is enough to prove that $\psi(a, T)$ $\cap \psi(b, T)=\phi$.

Suppose that $[l, k] \in \psi(a, T) \cap \psi(b, T)$ then:

$$
\left\{\begin{array}{c}
{[l, k] \in \psi(a, T) \rightarrow[l, k]=[a, k] \rightarrow(l, k) \sim(a, k)} \\
\rightarrow \exists e \in E(S), k \in E_{e}, a e=l e \\
{[l, k] \in \psi(b, T) \rightarrow[l, k]=[b, k] \rightarrow(l, k) \sim(b, k)} \\
\rightarrow \exists f \in E(S), k \in E_{f}, b f=l f
\end{array}\right.
$$

Since ef $\in E(S)$ and $e f=f e,\left(k \in E_{e} \cap E_{e f}\right)$, it can be replaced $e$ and $f$ with ef and finally we have:

$$
a e f=l e f, l e f=l f e=b f e=b e f
$$

Therefore we can find an element $e \in E(S)$ such that $\quad k \in E_{e}, a e=l e, l e=b e$. So $(l e)^{*}(l e)=a e l^{*} l e$ $=l e l^{*} l e=l l^{*} l e e=l e$, then $l e \leq a$, and similary $l e \leq b$, since $h=a \wedge b$ thus $l e \leq h$, then $l e=l e h^{*} h$, hence $l^{*} l e=l^{*} l e h^{*} h \leq h^{*} h$, and finally

$$
k \in E_{l^{*} l} \cap E_{e}=E_{l^{*} l e} \subseteq E_{h^{*} h}
$$

But $k \in T$ that is contradicts.

Definition 2.2 A zero in an inverse semigroup $S$ is an element $0 \in S$ such that:

$$
\text { oa }=a 0=0 \forall a \in S
$$

Definition 2.3 An inverse semigroup $S$ with zero is said to be $E^{*}$ - unitary if for every $e, a \in S$ one has that $e^{2} \neq e \leq a \Rightarrow a^{2}=a$.

In other words, if an element dominates a nonzero idempotent then that element itself is an idempotent.

Proposition 2.4 If $S$ is a $E^{*}$ - unitary inverse semigroup and $a, b$ belong to the defined semigroup $S$ such that $a^{*} a=b^{*} b$ and $a e=b e$ for some nonzero idempotent $e \leq a^{*} a$ then $a=b$.

Proof: We define $x=a e a^{*}$. So $x$ is nonzero idempotent because:

$$
e \leq a^{*} a \Rightarrow e=\left(a^{*} a\right)^{*}\left(a^{*} a\right) e=e a^{*} a a^{*} a
$$

Then $e=a^{*} a e a^{*} a$ (because of the ability of idempotent elements for being commute) and we have

$$
b a^{*} x=b a^{*} a e a^{*}=b b^{*} b e a^{*}=b e a^{*}=a e a^{*}=x .
$$

Therefore, we have $x \leq b a^{*}$. Since $S$ is a $E^{*}-$ unitary which implies that $b a^{*}$ is idempotent. Then $b a^{*}=\left(b a^{*}\right)^{*}=a b^{*}$ so $a b^{*}$ is idempotent as well.

But, we have

$$
b b^{*}=b b^{*} b b^{*}=b a^{*} a b^{*}=a b^{*} b a^{*}=a a^{*} a a^{*}=a a^{*}
$$

Setting $y=b a^{*} b$, we have that

$$
y^{*} y=b^{*} a b^{*} b a^{*} b=b^{*} a a^{*} a a^{*} b=b^{*} a a^{*} b=b^{*} b b^{*} b=b^{*} b
$$

Also $y^{*} y=a^{*} a$, while

$$
b=b b^{*} b=b y^{*} y, \text { and } a=a a^{*} a=a y^{*} y,
$$

So it is enough to prove that $y^{*}=a y^{*}$. We have

$$
a y^{*}=a b^{*} a b^{*}=a b^{*}=b a^{*}=b b^{*} b a^{*}=b b^{*} a b^{*}=b y^{*}
$$

In what follows we give the main result of this paper.

Theorem 2.5 In condition that $S$ is a $E^{*}$ - unitary inverse semigroup with zero, then can be appeared as a semilattice.

Proof: For proving the above theorem it is necessary to show that $a \wedge b$ exists for every $a, b \in S$. If there is not nonzero $h \in S$ such that $h \leq a, b$, it is obvious that 
$a \wedge b=0$ and it can be satisfied for the proof.

For doing this we can assume that there is a nonzero $h \in S$ in which $h \leq a, b$. Our claim is that $a b^{*} b=b a^{*} a$.

Suppose that $k=a^{*} a b^{*} b$ and considering to our assumption $\left(h^{*} h \leq a^{*} a, b^{*} b\right)$, we have $h^{*} h \leq k$.

Substituting $x=a k$ and $y=b k$,

$$
\left\{\begin{array}{l}
x^{*} x=k a^{*} a k=k^{2}=k \\
y^{*} y=k b^{*} b k=k^{2}=k
\end{array} \Rightarrow x^{*} x=y^{*} y\right.
$$

also

$$
x h^{*} h=a k h^{*} h=a h^{*} h=h=b h^{*} h=b k h^{*} h=y h^{*} h
$$

Using the proposition (2.4) $x=y$ will be achieved and so

$$
\begin{aligned}
& a b^{*} b=a a^{*} a b^{*} b=a k=x=y=b k \\
& =b a^{*} a b^{*} b=b\left(b^{*} b\right) a^{*} a=b a^{*} a
\end{aligned}
$$

and finally

$$
a b^{*} b=b a^{*} a
$$

By applying the above argument to $a^{*}, b^{*}, h^{*}$ and knowing that $h^{*} \neq 0$ and $h^{*} \leq a^{*}, b^{*}$ we have

$$
a^{*} b b^{*}=b^{*} a a^{*}
$$

so

$$
\left(a^{*} b b^{*}\right)^{*}=\left(b^{*} a a^{*}\right)^{*}
$$

and therefore Equation (1) can be modified to (2):

$$
b b^{*} a=a a^{*} b
$$

We have that $h \leq a, b$ then $h=a h^{*} h$ and $h=b h^{*} h$, then we can show that

$$
b^{*} a h^{*} h=b^{*} b h^{*} h=h^{*} h
$$

Since $S$ is a $E^{*}$ - unitary and $b^{*} a$ is dominated by $h^{*} h$, we have $\left(b^{*} a\right)^{2}=b^{*} a$. By applying the same reasoning to $a^{*}, b^{*}$ and $h^{*},\left(b a^{*}\right)^{2}=b a^{*}$ can be a result.

Thus

$$
\left\{\begin{array}{l}
\left(b^{*} a\right)^{*}=b^{*} a \\
\left(b a^{*}\right)^{*}=b a^{*}
\end{array}\right.
$$

and hence $a b^{*} b=b a^{*} b=b b^{*} a$

$$
a b^{*} b=b b^{*} a
$$

By combination of Equations (1) to (3), Equation (4) will be appeared.

$$
a b^{*} b=b a^{*} a=b b^{*} a=a a^{*} b
$$

At the end we try to prove that $a b^{*} b$ can satisfy the following condition

$$
h \leq a b^{*} b \leq a, b
$$

for every $h \in S$ such that $h \leq a, b$.

It is clear that $a b^{*} b \leq a, b$ and as defined before $k=a^{*} a b^{*} b$, then we have $h^{*} h \leq k$, and so

$$
h=a h^{*} h=a k h^{*} h=a a^{*} a b^{*} b h^{*} h=a b^{*} b h^{*} h=\left(a b^{*} b\right) h^{*} h
$$

Finally $h \leq a b^{*} b$. It means that $a b^{*} b$ is the join of $a$ and $b$ and this is the proof of theorem.

\section{References}

[1] M. B. Szendrei, “A Generalization of Mcalister's PTheorem for E-Unitary Regular Semigroups,” Acta Scientiarum Mathematicarum, Vol. 51, 1987, pp. 229-249.

[2] M. V. Lawson, "Inverse Semigroups: The Theory of Partial Symmetries,” Word Scientific, Singapore, 1998. doi:10.1142/9789812816689

[3] J. Renault, "A Groupoid Approach to C*-Algebra, Lecture Notes in Mathematics," 1st Edition, Springer-Verlang, Berlin, Vol. 793, 1980.

[4] J. M. Howie, "Fundamentals of Semigroup Theory," Clarendon Press, Oxford, 1995.

[5] H. Clifford and G. B. Preston, "The Algebric Theory of Semigroups," American Mathematical Society, United States, Vol. 1, 1961.

[6] N. Sieben, "C*-Crossed Products by Partial Actions and Actions of Inverse Semigroups," Journal of the Australian Mathematical Society, Series A, Vol. 63, No. 1, 1997, pp. 32-46.

[7] R. Exel, "Inverse Semigroups and Combinatorial C*Algebras," Bulletin of the Brazilian Mathematical Society, New Series, Vol. 39, No. 2, 2008, pp. 191-313.

[8] M. V. Lawson, "Inverse Semigroups, The Theory of Partial Symmetries,” Word Scientific, Singapore, 1998. 